\title{
Räumliche Verteilung der Innovationsleistung in der Schweiz
}

\section{Marco Caprarese, Basel}

\section{Einleitung}

«Kein anderer Industriestaat wächst so langsam wie die Schweiz. Die Schwäche der Wirtschaft hat langfristige Folgen: Der Wohlstand sinkt» (ZANCHI 2004: 23). Obwohl solche und ähnliche Aussagen in den letzten paar Monaten aufgrund des weltweiten Konjunkturaufschwungs seltener wurden, ändert sich nichts daran, dass sich das reale Wachstum des Bruttoinlandprodukts (BIP) der Schweiz seit 1965 sukzessive verschlechtert hat und die Schweiz in den 1990er Jahren als einziges Land der Organisation für wirtschaftliche Zusammenarbeit und Entwicklung (OECD) ein negatives Wachstum verzeichnen musste (Hotz-HART et al. 2003: 14). Auch der vom Basler Wirtschaftsforschungsinstitut BAK Basel Economics herausgegebene International Benchmark Report 2002 zeichnet ein ähnliches Bild und verweist die Schweiz in einem Vergleich besonders dynamischer Regionen in Bezug auf die Wachstumsrate auf den vorletzten Platz (Hotz-HarT et al. 2003: 15). Findet die Schweiz keinen Weg aus ihrer Wachstumsschwäche heraus, so zeigt sich auch für die Zukunft kein deutlich besseres Bild. Eine Studie der Deutschen Bank prognostiziert der Schweiz im Zeitraum 2006 bis 2020 ein durchschnittliches reales BIP-Wachstum von $0.7 \%$ pro Jahr, ein Ergebnis, das sie auf Platz 32 der 34 untersuchten Länder verweist (SCHNEIDER 2005: 4).

Über den Ernst der Lage wird häufig diskutiert. Oftmals wird argumentiert, dass die Wachstumsschwäche überschätzt werde, da das BIP pro Kopf als Produktivitätsmass die falsche Grösse sei, um dies zu beurteilen. BoDMER (2005: 6) hat aber aufgezeigt, dass auch die Betrachtung zahlreicher weiterer Grössen (vgl. Bodmer 2005: 9) nicht viel am Befund einer Wachstumsschwäche ändert: Die Schweiz belegt in internationalen Vergleichen bezüglich Wachstum und Dynamik immer einen der hintersten Plätze (ders.: 9). Sie befindet sich zwar momentan (noch) in einer guten Position, diese bröckelt aber zunehmends ab. Doch was kann oder soll die Schweiz machen, um diesem Trend zu begegnen und entgegenzuwirken? Und, ist es überhaupt nötig, nach immer mehr Wachstum zu streben? Geht es uns nicht bereits gut genug?

Was die Frage nach der Notwendigkeit des Wachstums für die Schweiz betrifft, so ist festzuhalten, dass es dabei nicht darum geht, ob der erarbeitete Wohlstand einfach gehalten oder noch gesteigert werden soll. Ohne Wachstum ist ein Beibehalten des aktuellen Niveaus kaum möglich. Die Gründe hierfür sind darin zu suchen, dass schwach wachsende Länder in der Regel im Standortwettbewerb an Attraktivität verlieren, grosse Probleme haben, die Staatsquote zu begrenzen, und Schwierigeiten mit der Alterssicherung bekommen. Erfahrungen aus anderen Ländern haben gezeigt, dass in stagnierenden Volkswirtschaften die sozialen Spannungen zunehmen und die Anstrengungen der verschiedenen Interessengruppen sich mehr auf das Verteilen des Kuchens als auf dessen Produktion konzentrieren (auch rent-seeking genannt), was die Situation weiter verschärft (SchWARZ 2004: 21).

Bezüglich der Massnahmen, um dieser Wachstumsschwäche zu begegnen, sind sich die Expertinnen und Experten meist einig: Da eine Steigerung der geleisteten Arbeitszeit in der Schweiz aufgrund des im internationalen Vergleich bereits hohen Niveaus kaum in Frage kommt, bleibt die Erhöhung der Produktivität als einzige Option für die Schweiz. Neue Technologien und Innovationen werden als die dafür geeigneten Mittel angeführt und deshalb als «Hauptmotoren» des Wachstums bezeichnet (Hotz-HaRT et al. 2003: 16).

Um geeignete Massnahmen zur Erhöhung der Innovationsleistung $\mathrm{zu}$ erarbeiten und $\mathrm{zu}$ implementieren, sind genaue Kenntnisse über die aktuelle Innovationsleistung und über im Verlaufe der Innovationserstellung auftretende Probleme nötig. Untersuchungen, welche sich dieser Problematik angenommen haben, existieren auf nationaler Ebene. Regionale Unterschiede bezüglich der Innovationsleistung gehen dabei allerdings unter. Für eine wirkungsvolle Politik sind diesbezügliche Kenntnisse jedoch nützlich. Die vorliegende Untersuchung hat deshalb zum Ziel, die Innovationsleistung der Schweiz auf einer subnationalen Ebene auszuwerten und zu untersuchen, ob in Bezug auf die Innovationsleistung regionale Unterschiede bestehen, dies sowohl für die gesamte Innovationsleistung als auch für die Innovationsleistung der einzelnen Phasen des Innovationsprozesses (siehe weiter unten).

\section{Methodisches Vorgehen}

\subsection{Definition von Innovationsindikatoren}

Um der Frage nach der Verteilung der Innovationsleistung nachzugehen, werden in einem ersten Schritt verschiedene Innovationsindikatoren definiert. Dazu wird auf das Konzept des Nationalen Innovationssy- 


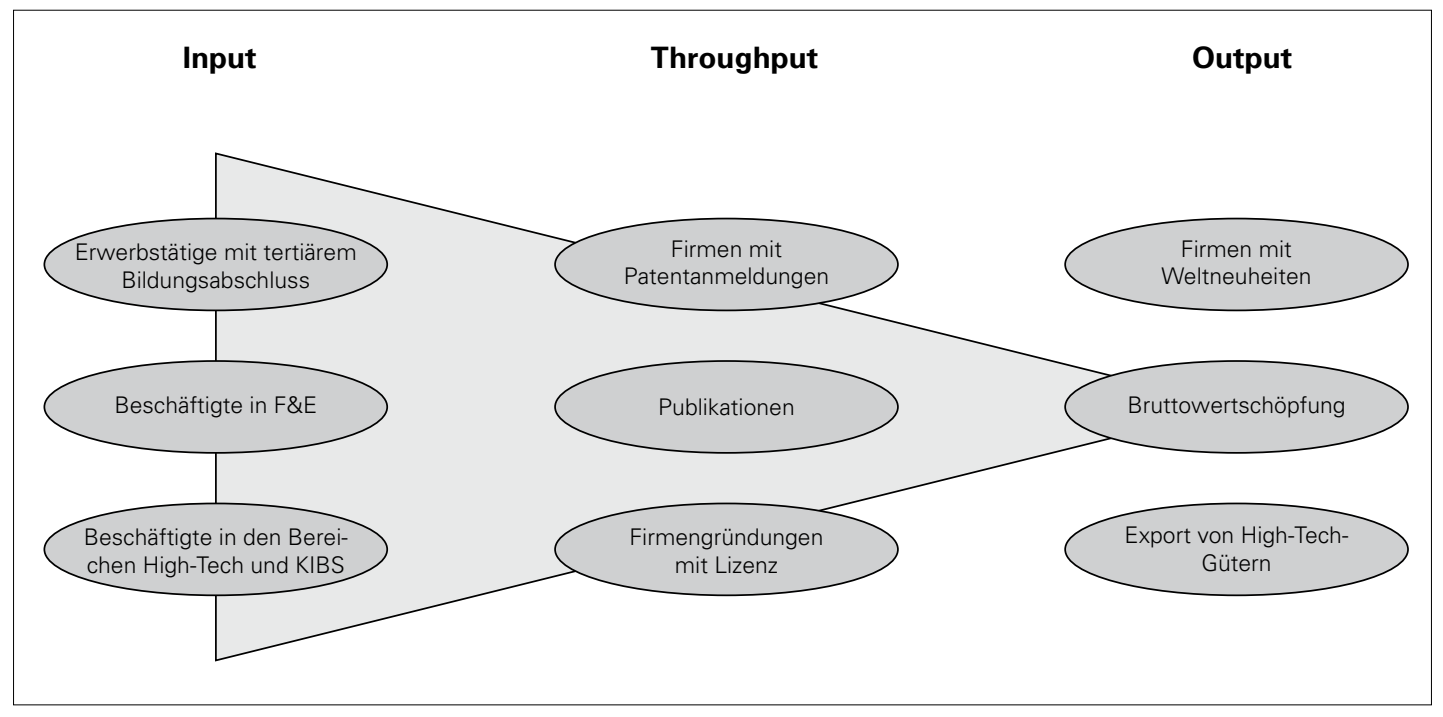

Abb. 1: Phasen des Innovationsprozesses und Beispiele zugeordneter Indikatoren Phases of the innovation process and examples of assigned indicators Phases du processus d'innovation et exemples d'indicateurs attribués

stems zurückgegriffen. Ziel ist es, die Innovationsleistung der verschiedenen ins Innovationssystem eingebundenen Akteure zu messen. Die Akteure werden hierzu in die fünf Grobkategorien Unternehmen, Bildungsinstitute, Forschungs- und Entwicklungs- (F\&E) Organisationen, Staat und Kantone sowie Finanzinstitutionen eingeteilt. Um bei der Erhebung eine gewisse Systematik zu gewährleisten, soll die Messung der Innovationsleistung dieser Akteure an verschiedenen Punkten des Innovationsprozesses erfolgen. In Anlehnung an DüMmLER (2005:4) wird dazu der Innovationsprozess als Basis genommen und in drei Teile gegliedert (vgl. Abbildung 1):

- Der erste Teil repräsentiert die Input-Phase des Innovationsprozesses. Diese Phase wird durch Indikatoren beschrieben, die den Input an Ressourcen in den Innovationsprozess aufzeigen, so zum Beispiel Indikatoren zum Humankapital und zur Forschungs intensität.

- Der an die Input-Phase anschliessende Teil des Innovationsprozesses wird beschrieben durch Indikatoren wie Publikationen, Patentanmeldungen oder auf Forschungsergebnissen beruhende Firmengründungen. Diese Phase wird Throughput-Phase genannt.

- Der Output des Innovationsprozesses wird durch Indikatoren wie «Bruttowertschöpfung», «Export von Hightech-Gütern» oder «Firmen mit Weltneuheiten» gemessen.
Die Wahl des für die Untersuchung verwendeten Regionalisierungsgrades hat datentechnische Gründe. Obwohl eine eher funktionale Abgrenzung erwünscht gewesen wäre, fiel die Wahl aus folgenden Gründen auf die politisch-administrativen Kantonsgrenzen:

- Ein Teil der Daten (zum Beispiel Zahlen zum Exportvolumen) sind lediglich auf Kantonsebene verfügbar.

- Die für die Umrechnung der Branchenwerte erforderliche Annahme homogener Unternehmen (siehe weiter unten) lässt sich auf Einzelbetriebsebene kaum aufrechterhalten. Es muss deshalb eine Gebietseinheit gewählt werden, die sicherstellt, dass darin eine genügend grosse Anzahl Betriebe enthalten ist. Da zum Beispiel MS-Regionen (MS $=m o b i-$ lité spatiale, vgl. DüMMLER et al. 2004: 30) dies nicht gewährleisten können, und Metropolitanräume oder Agglomerationen sich nicht flächendeckend über das gesamte Gebiet der Schweiz erstrecken, fiel die Wahl schliesslich auf den Kanton als Untersuchungseinheit.

Die Möglichkeiten bei der Wahl der Indikatoren werden durch die Verfügbarkeit geeigneter Datensätze beziehungsweise deren Aggregationsniveau stark limitiert, denn in der Schweiz durchgeführte Innovationsstudien werden meist auf der Ebene «Nation» durchgeführt, weshalb die dabei erhobenen Daten oftmals nicht regionalisierbar sind. 


\begin{tabular}{|ll|}
\hline Phase & Indikator \\
\hline Input-Phase & $\begin{array}{l}\text { Abschlüsse auf Tertiärstufe im technischen, naturwissenschaftlichen und } \\
\text { medizinischen Bereich } \\
\text { Erwerbstätige mit tertiärem Bildungsabschluss } \\
\text { Beschäftigte in den Bereichen High-Tech und KIBS } \\
\text { Anteil hochqualifizierten Personals } \\
\text { Beschäftigte in F\&E } \\
\text { Firmen mit F\&E-Kooperationen } \\
\text { Firmengründungen mit Lizenz } \\
\text { Publikationen } \\
\text { Firmen mit Patentanmeldungen } \\
\text { Neugründungen in den Bereichen High-Tech und KIBS } \\
\text { Firmen mit Exportaktivitäten } \\
\text { Firmen mit Produkt- und/oder Prozessinnovationen } \\
\text { Firmen mit Weltneuheiten } \\
\text { Export von High-Tech-Gütern } \\
\text { Bruttowertschöpfung }\end{array}$ \\
\hline Output-Phase & \\
\hline
\end{tabular}

Tab. 1: Innovationsindikatoren

Indicators of innovation

Indicateurs d'innovation

Einige der verwendeten Datensätze liegen zwar räumlich betrachtet ebenfalls auf der Aggregationsstufe «Nation» vor, lassen sich aber auch nach Branchen (in der Regel NOGA-Klassen - Nomenclature générale des activités économiques) aufschlüsseln, so zum Beispiel die Daten der Konjunkturforschungsstelle der Eidgenössischen Technischen Hochschule Zürich (KOF). Um solche Daten trotz ihres nationalen Bezuges für die vorliegende Analyse verwenden zu können, müssen sie unter Zuhilfenahme der Betriebszählungsdaten 2001 des Bundesamtes für Statistik (BfS) auf ein tieferes räumliches Aggregationsniveau gebracht werden. Gemäss BfS sind Klassen mit weniger als fünf Beobachtungen aus statistischen Gründen kritisch. Die Umrechnung erfolgt deshalb auf NOGA2-Stufe, da nur auf dieser Stufe sichergestellt werden kann, dass der grösste Teil der NOGA-Klassen mindestens fünf Beobachtungen aufweist. Klassen, die auch auf NOGA2-Stufe weniger als fünf Beobachtungen aufweisen, werden unter inhaltlichen Aspekten mit anderen Klassen vereint, so dass zum Schluss jede Klasse über mindestens fünf Beobachtungen verfügt. Zur Umrechnung der nationalen Branchendaten auf Kantonsniveau wird in einem ersten Schritt aus den Betriebszählungsdaten für jeden Kanton die Anzahl der in den verschiedenen Branchen beschäftigten Personen (Vollzeitäquivalente) ermittelt, in einem zweiten Schritt wird für jeden Kanton der auf ihn fallende Beschäftigungsanteil einer Branche bestimmt. Mit Hilfe dieser Beschäftigungsanteile werden die auf Branchenebene vorliegenden Daten auf die Kantone umgerechnet, das heisst der Indikatorwert, der einem Kanton zugewiesen wird, hängt direkt von den Beschäftigungsanteilen ab. Ein solches Vorgehen bedingt aber die Annahme homogener Unternehmen, das heisst, es wird davon ausgegangen, dass sich die einzelnen Unternehmen einer Branche in Bezug auf den durch den Indikator beschriebenen Sachverhalt nicht unterscheiden.

In die weitergehende Untersuchung fliessen 15 Indikatoren ein, wovon sich sechs auf die Input-Phase, vier auf die Throughput-Phase und fünf auf die Output-Phase beziehen. Um die Vergleichbarkeit sicherzustellen, wird bei allen Indikatoren immer der Zeitraum 2001 bis 2002 betrachtet, je nachdem, in welchem Jahr die entsprechenden Erhebungen durchgeführt wurden. Die 15 verwendeten Indikatoren sind in Tabelle 1 aufgeführt.

\subsection{Berechnung der Phasenindizes und des Gesamt- indexes}

Die in Tabelle 1 vorgestellten Indikatoren eignen sich zwar, die verschiedenen Teilaspekte des Innovationsprozesses und somit der Innovationsleistung $\mathrm{zu}$ erfassen, um sich ein Bild über die gesamthafte Inno- 
vationsleistung einer Region zu verschaffen, sind sie allerdings zu zahlreich. Ziel des nächsten Schrittes ist es deshalb, die Anzahl der Indikatoren auf einige wenige beziehungsweise auf einen einzigen Indikator zu reduzieren. Dazu stehen grundsätzlich zwei Verfahren zur Auswahl: Zum einen kann eine solche Reduktion der Variablen durch eine Faktoranalyse erreicht werden, zum anderen können sogenannte zusammengesetzte Indizes berechnet werden. Während eine Faktoranalyse die Variablen aufgrund der Korrelationskoeffizienten zu wenigen Faktoren reduziert, kann die Reduktion der Anzahl Variablen zu «zusammengesetzten» Indizes anhand rein inhaltlicher Gesichtspunkte erfolgen. Um die Frage nach der regionalen Gliederung der verschiedenen Phasen des Innovationsprozesses beantworten zu können, scheint eine Variablenreduktion unter Zuhilfenahme einer Faktoranalyse wenig sinnvoll, so dass im Folgenden in einem ersten Schritt drei «zusammengesetzte» Indizes berechnet werden. Die Variablen werden so zusammengefasst, dass jeder Index eine Phase des Innovationsprozesses repräsentiert (Input-, Throughput- und Output-Phase).

Wegen ihrer im Vergleich zu anderen Methoden relativ einfachen Berechnung ist der Gebrauch «zusammengesetzter» Indizes weit verbreitet. Generell stellen sich bei ihrer Berechnung drei Herausforderungen:

1. Welches Gewicht soll jedem einzelnen Subindikator beigemessen werden?

2. Wie sollen die verschiedenen Messgrösseneinheiten am besten auf ein einheitliches Niveau gebracht werden?

3. Wie kann trotz intervallskalierter Daten erreicht werden, dass Ausreisser nicht überbewertet werden und die Ergebnisse zu stark beeinflussen?

Arundel et al. (2002) sowie Hollanders \& ArunDEL (2004) haben sich dieser Problematik im Rahmen ihrer Arbeit zum European Innovation Scoreboard angenommen und fünf Varianten präsentiert, um verschiedene Messgrösseneinheiten auf ein einheitliches Niveau zu bringen. Für die Berechnung der «zusammengesetzten» Indizes wird im Rahmen dieser Arbeit folgende Methode verwendet, wobei, wie weiter unten beschrieben, auf eine Gewichtung verzichtet wird:

$$
\mathrm{Cl}_{\mathrm{i}}=\frac{\sum_{\mathrm{j}=1}^{\mathrm{m}} \mathrm{q}_{\mathrm{j}} \mathrm{y}_{\mathrm{ij}}}{\sum_{\mathrm{j}=1}^{\mathrm{m}} \mathrm{q}_{\mathrm{j}}} \text {, wobei } \mathrm{y}_{\mathrm{ij}}=\frac{\mathrm{x}_{\mathrm{ij}}}{\max _{\mathrm{i}} \mathrm{x}_{\mathrm{ij}}}
$$

wobei: $\mathrm{x}_{\mathrm{ij}}=$ Wert des Indikators $\mathrm{j}$ für den Kanton $\mathrm{i}$

$\mathrm{q}_{\mathrm{j}}=$ Gewichtung des Indikators $\mathrm{j}$

$\mathrm{y}_{\mathrm{ij}}=$ Wert des transformierten Indikators $\mathrm{j}$ für den Kanton i
Der Grund für die Wahl der obigen Formel liegt darin, dass sie sicherstellt, dass

1. alle in den Index einfliessenden Variablen nach der Transformation zwischen 0 und 1 verteilt sind. Demjenigen Kanton mit der höchsten gemessenen Ausprägung im betreffenden Indikator, kommt dabei der Wert 1 zu. Die Werte der anderen Kantone stellen nach der Transformation die Anteile bezogen auf denjenigen Kanton mit der besten Performance dar.

2. kein Informationsverlust aufgrund der Transformation auf ein tieferes Skalenniveau in Kauf genommen werden muss.

Aus den transformierten Indikatoren werden nun die verschiedenen Indizes für die Phasen des Innovationsprozesses sowie ein Index für die gesamte Innovationsleistung berechnet:

- Zur Berechnung der Indizes der einzelnen Phasen des Innovationsprozesses wird aus den transformierten Indikatorenwerten das arithmetische Mittel gebildet. Auf eine Gewichtung wird bewusst verzichtet, da für eine sinnvolle Schätzung der einzelnen Gewichte eine Zielvariable notwenig wäre. Unter Kenntnis der Zielvariablen wäre es möglich, anhand einer Regressionsanalyse die Gewichte, mit denen die einzelnen Indikatoren auf die Zielvariable wirken, zu berechnen. Diese Zielvariable ist hier aber unbekannt, da sie eigentlich das Ziel der ganzen Untersuchung darstellt und durch den «zusammengesetzten» Index ausgedrückt wird (ARUNDEL et al. 2002: 11).

- Der «Gesamtindex» wird aus den Phasenindizes gebildet, indem aus diesen drei Indizes wiederum das arithmetische Mittel berechnet wird. Bei diesem Vorgehen kann der Einwand eingebracht werden, dass dadurch, dass die einzelnen Phasenindizes je aus einer unterschiedlichen Anzahl Indikatoren gebildet wurden, die ursprünglichen Indikatoren nun mit unterschiedlicher Gewichtung in den Gesamtindex einfliessen. Der Grund für dieses Vorgehen liegt aber darin, dass die Auswahl der Indikatoren, wie weiter oben bereits erwähnt, stark durch die Verfügbarkeit geeigneter Datensätze eingeschränkt wird, so dass es nicht möglich ist, für alle Phasenindizes die gleiche Anzahl Indikatoren zu definieren. Da das Ziel bei den Phasenindizes aber auch darin liegt, diese möglichst genau und umfassend zu erstellen, werden bei jedem einzelnen Phasenindex alle verfügbaren Indikatoren berücksichtigt. Für die Errechnung des Gesamtindexes wird nun keine Gewichtung der Phasenindizes vorgenommen, da, wie schon bei der Erstellung der Phasenindizes, die einzelnen Gewichte ohne Kenntnis der Zielvariablen nicht errechnet werden können. 


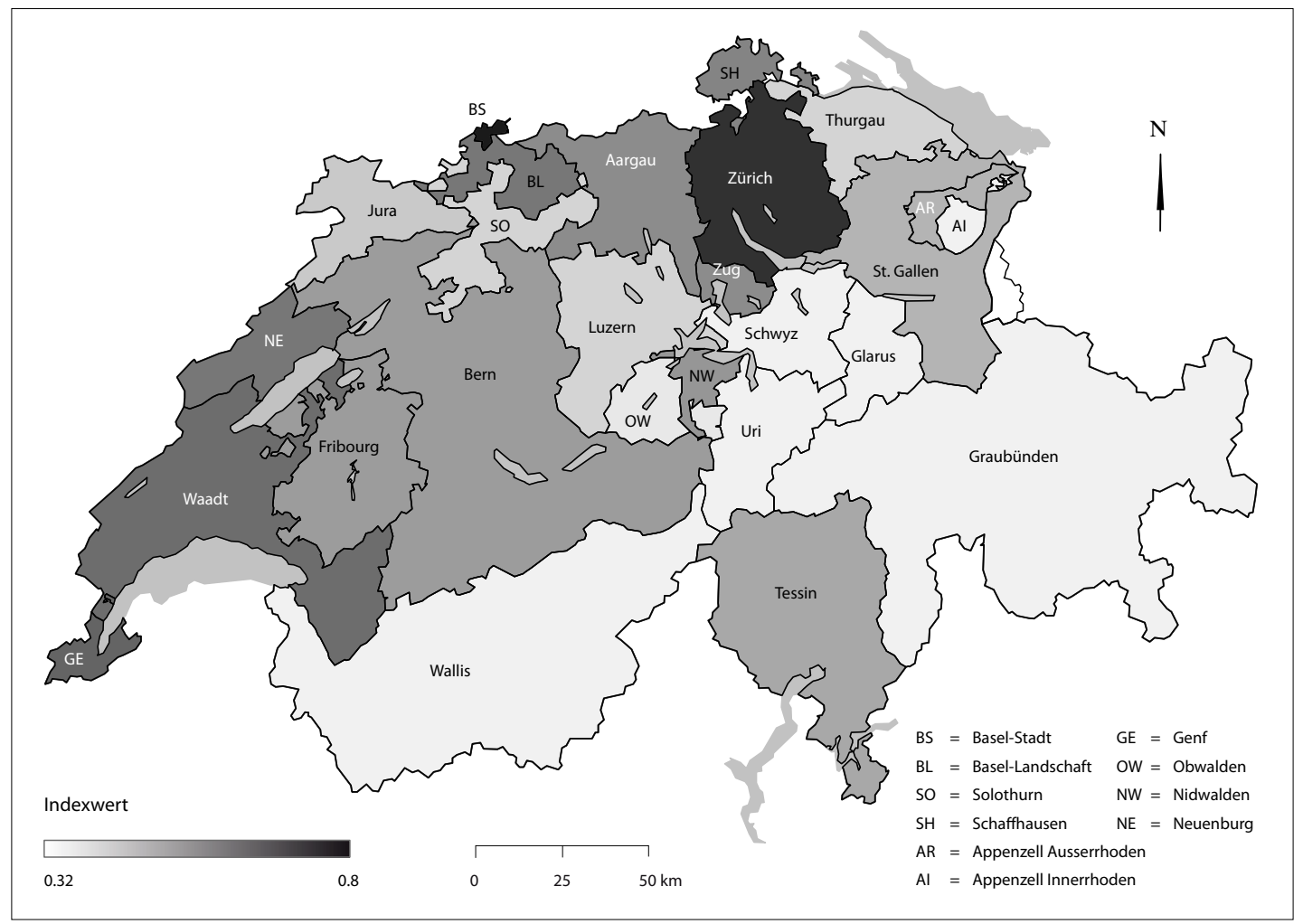

Abb. 2: Innovationsindex der Input-Phase in den Kantonen der Schweiz Index of innovation for the input phase, by canton of Switzerland Index d'innovation de la phase d'input dans les cantons suisses Quelle: eigene Darstellung

\section{Räumliche Verteilung der Innovationsleistung}

Um der Frage nachzugehen, ob in der Schweiz eine regionale Gliederung der verschiedenen Phasen des Innovationsprozesses ersichtlich ist, wurden aus den im vorangegangenen Teilkapitel vorgestellten Indikatoren drei «zusammengesetzte» Indizes gebildet, welche die einzelnen Phasen des Innovationsprozesses (Input-Phase, Throughput-Phase und Output-Phase) repräsentieren. Diese werden nachfolgend vorgestellt.

\subsection{Input-Phase}

Der die Input-Phase repräsentierende Index setzt sich aus sechs einzelnen Indikatoren zusammen (siehe Tabelle 1). Sein möglicher Wertebereich reicht von 0 bis 1, wobei 1 das Maximum darstellt.

Die Werte des Input-Indexes reichen von 0.32 bis 0.8 . Ein Blick auf die Karte in Abbildung 2 zeigt, dass die
Alpenkantone generell schlechter abschneiden als die Mittellandkantone. Im Mittelland und in der Nordwestschweiz heben sich die Regionen Basel, Zürich und Genfersee-Neuenburg deutlich vom Rest ab.

Unter den Top-Five-Kantonen befinden sich die Kantone Basel-Stadt, Zürich, Genf, Waadt und Neuenburg. Betrachtet man die einzelnen Indikatoren, die zu diesem Ergebnis führten, so fällt auf, dass die Kantone Basel-Stadt, Zürich und Genf in mindestens einem dieser Input-Indikatoren auf dem ersten Platz zu finden sind:

- Der Kanton Basel-Stadt schneidet bei allen Indikatoren mit Ausnahme des Indikators «Abschlüsse auf Tertiärstufe im technischen, naturwissenschaftlichen und medizinischen Bereich» gut bis sehr gut $\mathrm{ab}$, in Bezug auf den Anteil Beschäftigter in den Bereichen High-Tech und Knowledge Intensive Business Services (KIBS) sowie in Bezug auf den 
Anteil hochqualifizierten Personals in den Unternehmen belegt er sogar den Spitzenplatz.

- Der Kanton Zürich schneidet vor allem bei den Indikatoren «Abschlüsse auf Tertiärstufe im technischen, naturwissenschaftlichen und medizinischen Bereich», «Beschäftigte in den Bereichen HighTech und KIBS» sowie «Anteil hochqualifizierten Personals» gut bis sehr gut ab. Das gute Ergebnis des Kantons Zürich in Bezug auf die vergebenen Diplome und Doktorentitel überrascht wenig, beheimatet er doch mit der Universität Zürich, der Eidgenössischen Technischen Hochschule (ETH) Zürich und der Zürcher Fachhochschule drei grosse Bildungsinstitutionen auf Tertiärstufe. Die Grösse dieser drei Institutionen wird auch daran deutlich, dass hier im Herbst 2005 rund 47'000 Studierende immatrikuliert waren, womit $28 \%$ aller Schweizer Hochschüler im Kanton Zürich studierten (FURGER 2005: 27). Im Gegensatz zum Kanton Basel-Stadt schneidet der Kanton Zürich allerdings hinsichtlich der Beschäftigten in F\&E schlecht ab (BS: 0.86; ZH: $0.37)$.

- Der Kanton Genf steht vor allem hinsichtlich des Bildungsniveaus der Arbeitsbevölkerung (Erwerbstätige mit tertiärem Bildungsabschluss), des Anteils Beschäftigter in den Bereichen High-Tech und KIBS sowie in Bezug auf den Anteil hochqualifizierten Personals in den Unternehmen gut bis sehr gut da. Eher mager ist das Resultat in Bezug auf die vergebenen Diplome und Doktorentitel (im Vergleich zu den Kantonen Zürich und Waadt) und hinsichtlich des Anteils F\&E-Beschäftigter.

Zusammenfassend kann festgehalten werden, dass in Bezug auf die Input-Phase vor allem die Kantone entlang des Alpenkamms schlecht abschneiden. Von den restlichen Kantonen rangieren diejenigen, in welchen die vier grossen Wirtschaftszentren des Landes (Zürich, Basel, Genf, Lausanne) liegen, klar vorne. Weiter stehen auch die Kantone Bern und Tessin nicht schlecht da. Der Grund hierfür ist darin zu suchen, dass beide Kantone über zahlreiche Hochschuleinrichtungen verfügen, was sich bezüglich der Input-Phase des Innovationsprozesses als klar leistungssteigernd auswirkt.

\subsection{Throughput-Phase}

Throughput-Indikatoren zeigen sozusagen das Zwischenergebnis im Innovationsprozess auf. Der die Throughput-Phase repräsentierende Index setzt sich aus vier Indikatoren zusammen (siehe Tabelle 1). Sein möglicher Wertebereich reicht von 0 bis 1 , wobei 1 das Maximum darstellt.

Die Werte des Throughput-Indexes bewegen sich zwischen 0.19 und 0.57 , sind also deutlich tiefer als diejenigen des Input-Indexes. Der Grund für die tieferen
Werte liegt zum Teil an der gewählten Methode, um die Indikatorenwerte auf ein einheitliches Niveau zu transformieren: Sie rechnet die Resultate der einzelnen Kantone jeweils in Anteile des Wertes des am besten abschneidenden Kantons um (vgl. Kapitel 2). Da sich bei den vier Throughput-Indikatoren meist ein oder zwei Kantone deutlich von den anderen Kantonen abheben, fallen die transformierten Werte der restlichen Kantone eher tief aus.

Die in Abbildung 3 dargestellte Karte zeigt, dass in Bezug auf die Throughput-Phase die Westschweiz (Region Genfersee-Neuenburg) und NordwestSchweiz (vor allem die Region Basel-Aargau) deutlich besser abschneiden als die übrigen Gebiete. Die TopFive in Bezug auf den Innovations-Throughput bilden die Kantone Aargau, Waadt, Basel-Stadt, Neuenburg und Genf:

- Beim Kanton Aargau geht das gute Resultat vor allem auf hohe Werte bei den Indikatoren «Firmenneugründungen mit Lizenz» und «Firmen mit $\mathrm{Pa}$ tentanmeldungen» zurück.

- Der Kanton Waadt verdankt seinen zweiten Platz auch dem guten Resultat in Bezug auf die Firmengründungen mit Lizenz pro 1000 Hochschulmitarbeiter sowie dem Umstand, dass er bei den anderen Indikatoren nirgends merklich schlechter abschnitt als die anderen Kantone.

- Massgebend für die hohen Werte des Kantons Basel-Stadt ist vor allem sein relativ zur Zahl der Angestellten im Hochschul- oder F\&E-Bereich hohes Publikationsvolumen.

- Die gute Rangierung des Kantons Neuenburg lässt sich zu einem grossen Teil mit seinem sehr guten Resultat in Bezug auf die Patentierungsaktivitäten der dort ansässigen Unternehmen erklären.

- Das gute Resultat des Kantons Genf geht wie beim Kanton Basel-Stadt zu einem grossen Teil auf das relativ zur Zahl der Angestellten im Hochschul- und F\&E-Bereich hohe Publikationsvolumen zurück.

Wie gezeigt, ist die Verteilung der Innovationsleistung bezüglich der Throughput-Phase wiederum nicht homogen über die Schweiz verteilt, sondern es bestehen regionale Unterschiede. Obwohl die Zentren der West- und der Nordwestschweiz klar in den vordersten Rängen zu finden sind und die Alpenkantone das Schlusslicht des Rankings bilden, kann nicht eindeutig gesagt werden, dass Zentren, bezogen auf die Throughput-Phase, innovativer sind als ländliche Regionen. Der Grund hierfür liegt darin, dass Zürich als grösster Wirtschaftsraum der Schweiz lediglich im Mittelfeld plaziert ist.

\subsection{Output-Phase}

Output-Indikatoren zeigen den Output des Innovationsprozesses auf, stellen also in einem gewissen 


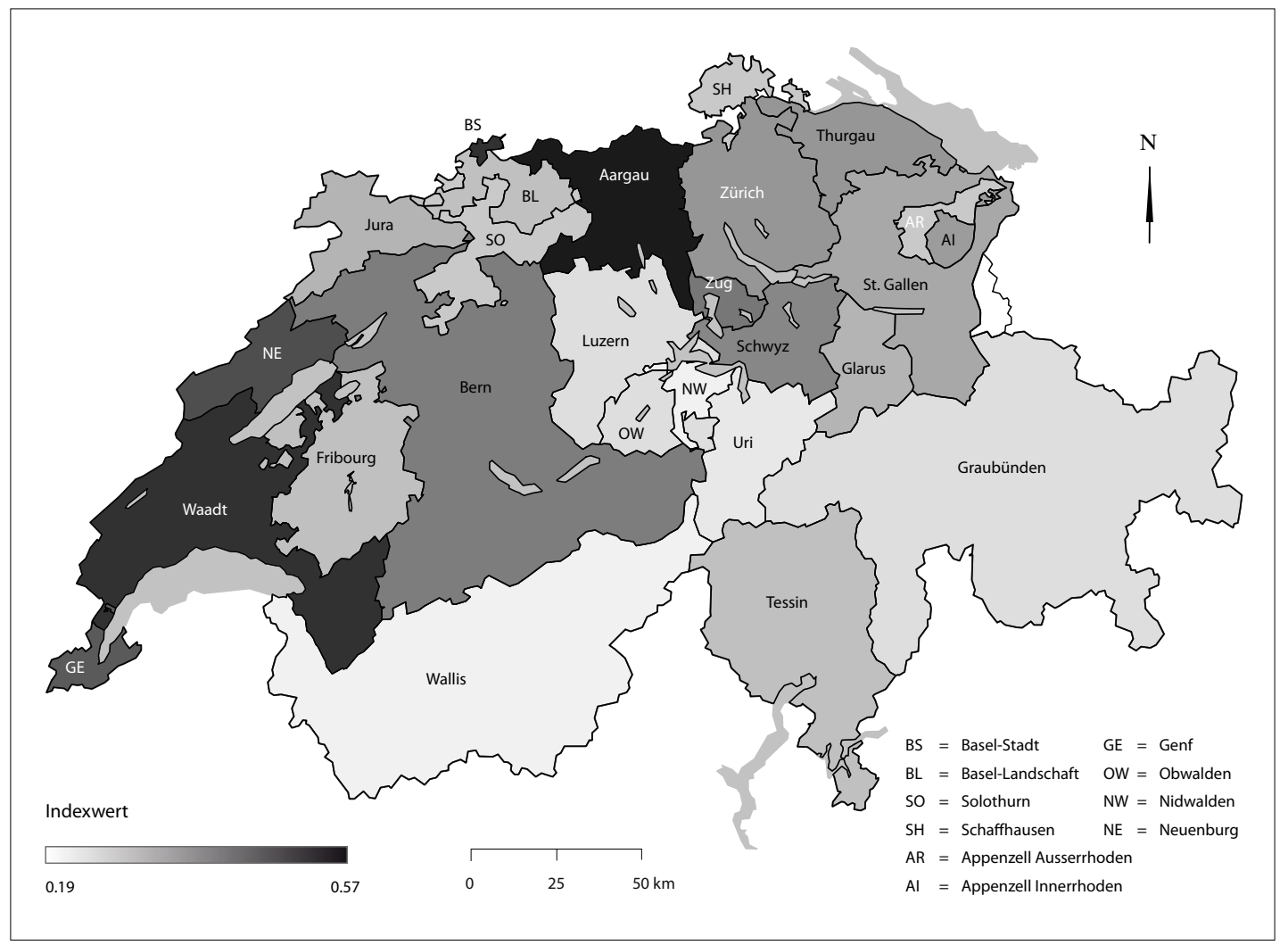

Abb. 3: Innovationsindex der Throughput-Phase in den Kantonen der Schweiz Index of innovation for the throughput phase, by canton of Switzerland Index d'innovation de la phase de throughput dans les cantons suisses Quelle: eigene Darstellung

Masse die Innovationen und ihren wirtschaftlichen Erfolg dar. Der die Output-Phase repräsentierende Index setzt sich aus fünf Output-Indikatoren zusammen (siehe Tabelle 1). Der mögliche Wertebereich des Output-Indexes reicht wie bei den beiden vorhergehenden Indizes von 0 bis 1 , wobei 1 wiederum das mögliche Wertemaximum darstellt.

Ein Blick auf die in Abbildung 4 dargestellte Karte zeigt, dass es im Falle des Output-Indexes nicht die städtischen Wirtschaftszentren sind, welche gut abschneiden, sondern eher die ländlichen Industriekantone. So ist unter den Top-Five-Kantonen diesmal keiner der Kantone mit einer der grösseren Städte zu finden. Am besten schnitt mit Abstand der Kanton Appenzell Ausserrhoden ab, gefolgt von den Kantonen Glarus, Jura, Uri und Freiburg. Das viel bessere Resultat der Industriekantone gegenüber den Dienstleistungskan- tonen lässt sich zu einem gewissen Teil damit erklären, dass ein Grossteil der Output-Indikatoren eher auf die Messung von Industrie-Output ausgerichtet ist. In den Kantonen Appenzell Innerrhoden und Glarus dürfte die Textilindustrie zu einem grossen Teil für das gute Resultat verantwortlich sein. Im Kanton Jura trägt die dort ansässige Uhrenindustrie sicherlich einen grossen Teil zum guten Ergebnis des Kantons bei.

Vergleicht man Abbildung 4 mit den Abbildungen 2 und 3, so zeigt sich, dass die räumliche Verteilung der auf die Output-Phase bezogenen Innovationsleistung weit homogener über die Schweiz verteilt ist, als die auf die Input- und auf die Throughput-Phase bezogene Innovationsleistung. Lässt man den Kanton Appenzell Ausserrhoden für einmal ausser Betracht, so zeigt sich, dass die Unterschiede zwischen den Kantonen nicht sehr gross sind. Dies verdeutlichen auch 


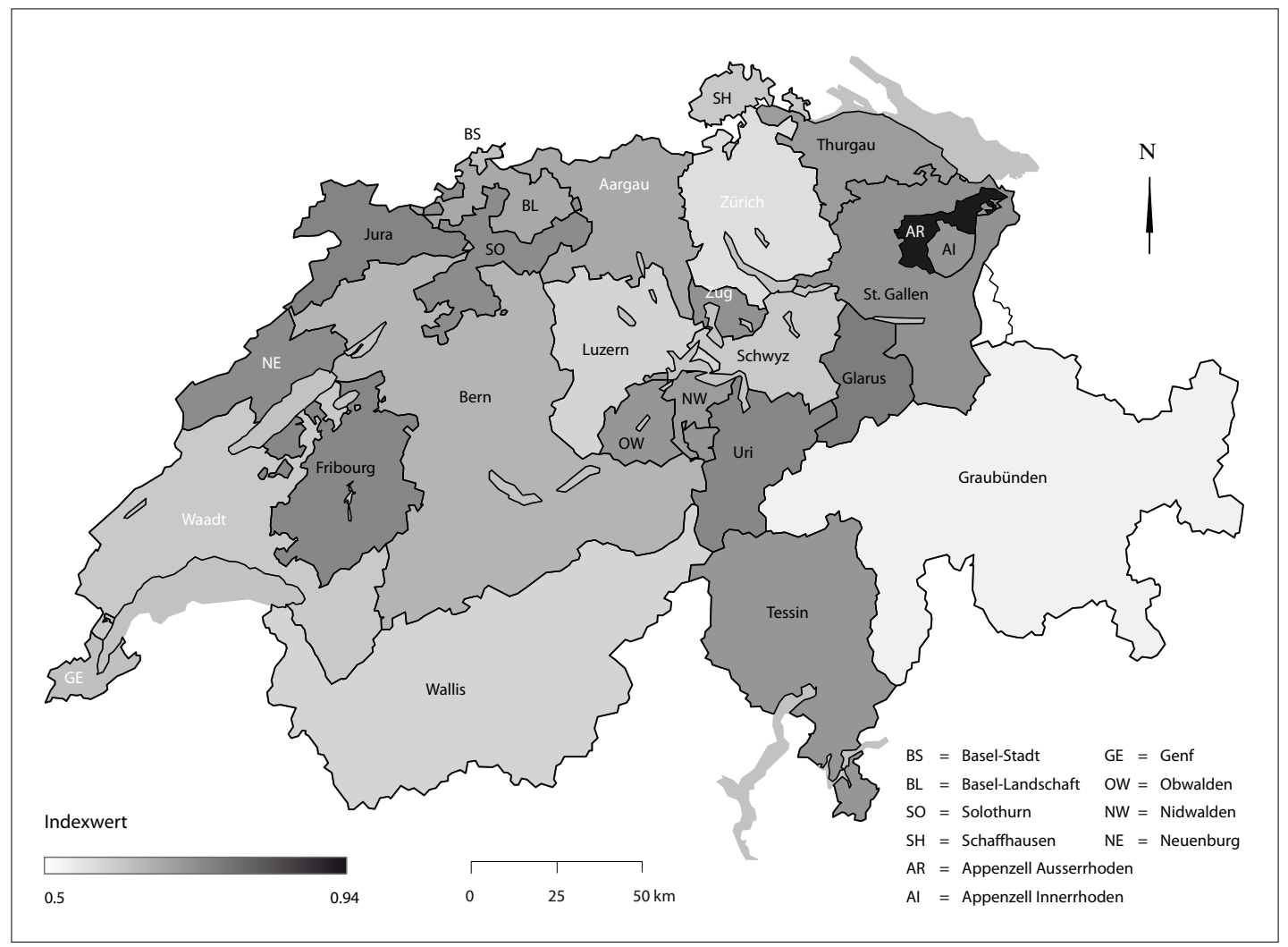

Abb. 4: Innovationsindex der Output-Phase in den Kantonen der Schweiz

Index of innovation for the output phase, by canton of Switzerland

Index d'innovation de la phase d'output dans les cantons suisses

Quelle: eigene Darstellung

die Indexwerte: Mit einem Unterschied von lediglich 0.26 zwischen dem zweitplazierten Kanton Glarus und dem letztplazierten Kanton Graubünden liegt die Variabilität deutlich unter derjenigen, welche in den anderen Phasen gemessenen werden konnte. Betrachtet man die Top-Five-Kantone, so fällt auf, dass die Wirtschaftszentren der Schweiz nicht darunter vertreten sind, sondern dass es sich bei diesen Kantonen eher um ländliche Industriekantone handelt.

Zusammenfassend kann festgehalten werden, dass zwar eine gewisse heterogene Verteilung der auf die Output-Phase des Innovationsprozesses bezogenen Innovationsleistung besteht, diese aber im Vergleich $\mathrm{zu}$ derjenigen anderer Phasen nicht besonders ausgeprägt ist. Weiter kann die Behauptung, dass Zentren hinsichtlich der auf die Output-Phase bezogenen Innovationsleistung innovativer sind als ländliche
Regionen, nicht aufrecht erhalten werden, da unter den Top-Five-Kantonen keines der Wirtschaftszentren des Landes vertreten ist. Es könnte im Gegenteil sogar vielmehr die Hypothese aufgestellt werden, dass der Output des Innovationsprozesses eher in den ländlichen Industriekantonen produziert wird als in den Dienstleistungszentren.

\subsection{Gesamthafte Innovationsleistung}

Um die Verteilung der gesamthaften Innovationsleistung zu untersuchen, wurden die drei Phasenindizes zu einem Gesamtindex vereint (vgl. Kapitel 2). Wie bei den drei Teilindizes bewegen sich die möglichen Werte des Indexes zwischen 0 und 1, wobei auch hier 1 wieder das mögliche Maximum darstellt.

Die Karte in Abbildung 5 stellt die Verteilung der Innovationsleistung graphisch dar. Die Werte des 


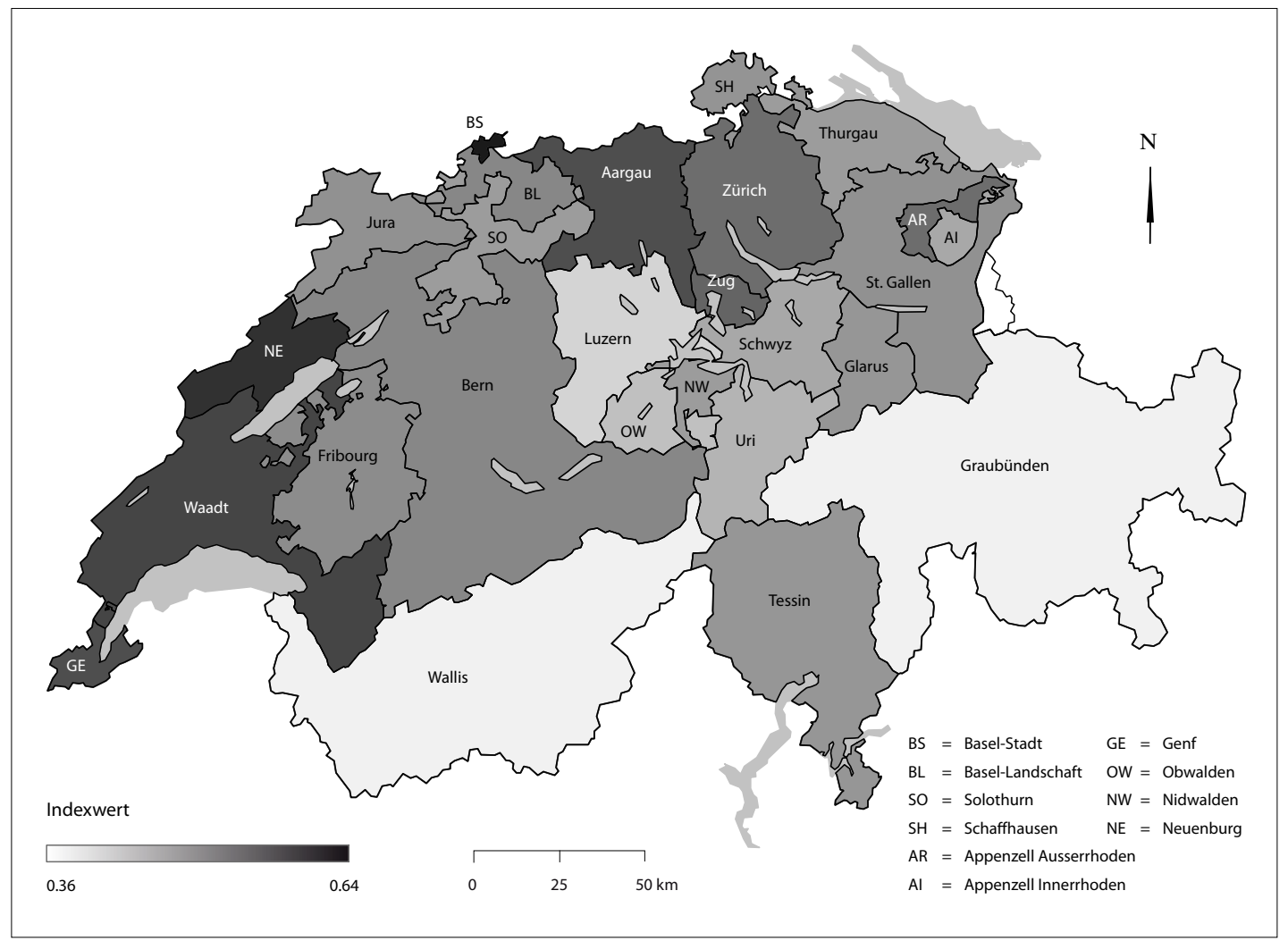

Abb. 5: Gesamtinnovationsindex in den Kantonen der Schweiz Overall index of innovation, by canton of Switzerland Index total d'innovation dans les cantons suisses Quelle: eigene Darstellung

Gesamtindexes bewegen sich zwischen 0.36 und 0.64. Klar ersichtlich ist, dass das Mittelland besser da steht als die Kantone entlang des Alpenkamms. Dies ist allerdings nicht sehr erstaunlich. Am schlechtesten schnitten die Kantone Graubünden und Wallis ab. Gute bis sehr gute Resultate erzielten die Region Basel-Aargau-Zürich-Zug sowie die Region Genfersee-Neuenburg. Das gute Resultat des Kantons Appenzell Ausserrhoden kann hauptsächlich auf das gute Gesamtbild beim Output-Index zurückgeführt werden.

\section{Fazit}

Die Meinung, Innovationen seien eine Sache der Zentren, nicht aber des eher ländlichen Raumes, ist weit verbreitet. Der ländliche Raum wird oftmals mit ganz anderen Attributen assoziiert wie zum Beispiel Erholung, Natur und Landwirtschaft. Dass Innovationen eher in den Zentren des Landes generiert werden, scheint sich auf einen ersten Blick sogar zu bestätigen. Wie in Kapitel 3.4 gezeigt, weisen Zentrumskantone gesamthaft betrachtet eine höhere Innovationsleistung auf als ländliche Kantone. Dieses Bild ändert sich aber, betrachtet man nicht die gesamthafte Innovationsleistung, sondern wird diese nach den einzelnen Phasen des Innovationsprozesses aufgeteilt. Dabei zeigt sich, dass der Innovations-Input und der Innovations-Throughput zwar oftmals in den Zentren generiert werden, der Innovations-Output aber zu einem guten Teil in den ländlichen Industriekantonen produziert wird. Der ländliche Raum ist also nicht per se der innovationsleere Raum, für welchen er oftmals gehalten wird. Dieser Befund soll nun aber nicht darüber hinwegtäuschen, dass weite Teile dieses 
ländlichen Raumes über ein Innovationsdefizit verfügen. Wie die Analyse gezeigt hat, ist dieses entlang des Alpenkammes am grössten.

\section{Literatur}

Arundel, A., Bordoy, C. \& H. Hollanders (2002): 2002 European innovation scoreboard. Methodology report (technical paper No 6). - Discussion Paper des «Innovations/SMEs Programme» der Europäischen Union. - Brüssel.

Bodmer, F. (2005): Hat die Schweiz ein Wachstumsproblem? - Präsentation am Wachstumskongress von Avenir Suisse vom 4.3.2005, Zürich.

Dümmler, P., Abegg, C., Kruse, C. \& A. Thierstein (2004): Standorte der innovativen Schweiz. Räumliche Veränderungsprozesse von High-Tech und Finanzdienstleistungen. - Neuchâtel: Bundesamt für Statistik (BfS).

DÜMMLER, P. (2005): Innovationsstandort Schweiz oder Innovation entstand dort. Wissensbasierte Cluster als Entwicklungschance. - Discussion Paper, Institut für Raum- und Landschaftsentwicklung, Fachbereich Raumordnung, ETH Zürich, Nr. 1-2005, Zürich.

Furger, M. (2005): Semesterstart für 47'000 Studierende. - In: Neue Zürcher Zeitung (NZZ), Nr. 248, 24.10.2005, Zürich: 27.

Hollanders, H. \& A. Arundel (2004): 2004 European innovation scoreboard. Methodology report. - Discussion Paper des «Innovations/SMEs Programme» der Europäischen Union. - Brüssel.

Hotz-Hart, B., Good, B., Küchler, C. \& A. ReuterHofer (2003): Innovation Schweiz. Herausforderungen für Wirtschaft und Politik. - Zürich: Rüegger.

SCHNEIDER, S. (2005): Globale Wachstumszentren 2020. Formel-G für 34 Volkswirtschaften. - Themen International: Economics, Nr. 313, 9.2. 2005, Frankfurt am Main: Deutsche Bank Research.

SchwARZ, G. (2004): Ein bisschen Wachstum ist nicht genug. - In: Neue Zürcher Zeitung (NZZ), Nr. 25, 31.1.2004, Zürich: 21.

ZANCHI, M. (2004): Wegen Stillstand weniger Wohlstand. - In: Tages-Anzeiger, Nr. 32, 9.2.2004, Zürich: 23.

\section{Zusammenfassung: Räumliche Verteilung der Innovationsleistung in der Schweiz}

Innovation und Wachstum sind eng miteinander verknüpft und wichtige Determinanten für den Wohlstand eines Landes. Angesichts der vor allem in den 1990er Jahren akuten Wachstumsschwäche der Schweiz wurde viel über Wirtschaftswachstum und Innovationsleistung diskutiert. Um geeignete Massnahmen zur Erhöhung der Innovationsleistung zu erarbeiten und zu implementieren, sind aber genaue Kenntnisse über die aktuelle Innovationsleistung erforderlich. Die vor- liegende Arbeit untersucht die Innovationsleistung der Schweiz auf einer subnationalen Ebene. Hierfür wurde der Innovationsprozess in drei Phasen aufgeteilt: Innovations-Input, -throughput und -output. Für jede dieser drei Phasen wurden Indikatoren definiert, welche die entsprechende Phase möglichst umfassend abbilden. Diese Indikatoren wurden anschliessend zu einem die Phase repräsentierenden Index zusammengefasst, und in einem letzten Schritt wurden diese drei Phasenindizes zu einem Gesamtindex addiert. Die Untersuchung hat gezeigt, dass die Zentren gesamthaft betrachtet innovativer sind als der ländliche Raum, dies aber nicht für alle Phasen des Innovationsprozesses und nicht für den gesamten ländlichen Raum gilt.

\section{Summary: Spatial distribution of innovation activities in Switzerland}

Innovation and economic growth are closely linked to each other. Together, they are considered an important determinant of a nation's wealth. In the light of weak economic growth in Switzerland in the 1990's, a good deal was spoken and written about these two indicators. In order to develop and implement appropriate actions for increasing innovation activity, accurate information about current activity in this area is essential. Thus, the paper presents an analysis of Switzerland's innovation activity at a subnational level. For this purpose, the innovation process was divided into three phases: innovation input, innovation throughput, and innovation output. A set of appropriate indicators for each phase was defined. A quantitative analysis of the indicators resulted in indices for each phase, the total of which is referred to as the overall index. The overall index was seen to shed light on an entity's total innovation activity. The study indicates that on the whole, the economic centres of the country are more innovative than rural regions. However, this was not the case for all phases of the innovation process or for all rural areas.

\section{Résumé: La distribution spatiale des activités innovantes en Suisse}

L'innovation et la croissance économique sont fortement liées entre elles et forment des déterminants importants pour la prospérité d'une nation. Compte tenu de la faiblesse de la croissance économique suisse, les politiciens et les médias de ce pays ont souvent discuté de ces deux thèmes. Afin de développer et d'instaurer des actions appropriées pour augmenter les activités innovantes, il est très important d'en avoir des informations exactes. Ce travail analyse les activités innovantes en Suisse au plan subnational. Pour cela, le processus d'innovation a été fractionné en trois phases qui correspondent à l'input, au throughput et à l'output d'innovation. Des indicateurs différents correspondant à ces phases ont ensuite été définis. Afin 
d'obtenir une mesure de chacune de ces phases, les indicateurs ont par la suite été fusionnés en trois indices. Finalement, ces trois indices ont été résumés en un index unique, qui représente les activités innovantes totales d'une entité spatiale. L'étude montre alors que les centres économiques du pays sont plus innovants que les régions rurales, mais que ce constat ne vaut ni pour toutes les phases du processus d'innovation ni pour toutes les régions rurales.

Dipl.-Geogr. Marco Caprarese, Geographisches Institut, Abteilung Humangeographie / Stadt- und Regionalforschung, Klingelbergstrasse 27, CH-4056 Basel, Schweiz.

e-mail:marco.caprarese@unibas.ch

Manuskripteingang/received/manuscrit entré le 21.5.2007

Annahme zum Druck/accepted for publication/accepté pour l'impression: 20.11.2007 